\title{
Adaptive Device-Free Passive Localization Coping with Dynamic Target Speed
}

\author{
Xiuyuan Zheng*, Jie Yang ${ }^{\dagger}$, Yingying Chen*, Yu Gan* \\ *Dept. of ECE, Stevens Institute of Technology \\ ${ }^{\dagger}$ Dept. of CSE, Oakland University \\ Castle Point on Hudson, Hoboken, NJ 07030 \\ \{xzheng1, yingying.chen,ygan\}@stevens.edu \\ Rochester, Michigan 48309 \\ yang@oakland.edu
}

\begin{abstract}
Device-free passive localization enables locating targets (e.g., intruders or victims) that do not carry any radio devices nor do they actively participate in the wireless localization process. This is because the wireless environments will get affected when people move into the area, which result in the changes of Received Signal Strength (RSS) of the wireless links. In this paper, we first show that the localization performance degrades significantly when people are moving in dynamic speeds. This is because existing studies in device-free passive localization system have an implicit assumption that the target is moving at a constant speed, which is not always true in practical scenarios. To cope with targets moving with dynamic speeds, we propose an adaptive speed change detection framework including three components: speed change detection, determination of time-window size and adaptive localization. Two speed change detection schemes have been developed to capture the changes of moving speed and adjust the time-window size adaptively to facilitate effective localization. We demonstrate that our framework is flexible to work with any device-free localization method using signal strength. Results from the real experiments confirm that our approach has over $30 \%$ improvement on both median and max localization error, under dynamically changing speed of the target.
\end{abstract}

\section{INTRODUCTION}

Device-free passive localization is an emerging technology to locate people without attaching any radio device to them in pervasive wireless environments. It has broad applications [1][3] in intrusion detection in industrial facilities for asset protection, identification of people trapped in a fire building during emergency evacuation, elder care, and battlefield protection. In these applications, we do not expect people to carry any radio devices, and thus the traditional localization techniques [4], which require wireless devices attached to people to emit wireless signals to assist localization process, are not applicable. With the widespread deployment of wireless devices, it is possible to capture the wireless environmental changes caused by people or intruders who move into the wireless environments. Therefore, device-free passive localization has drawn much attention recently for motion detection and target localization in pervasive wireless environments.

More than one modalities of wireless signal measurements, including Received Signal Strength (RSS) [5], channel impulse response in Ultra Wide Band (UWB) [6], polarization [7], etc, have been utilized to facilitate device-free localization. Among all these modalities, RSS is especially attractive as the RSS readings are readily available in the existing wireless infrastructure and presents tremendous cost savings. Thus, there has been increasing interest in employing RSS for device-free passive localization recently [5] .

Existing RSS-based device-free passive localization systems mainly rely on the changes of variance of RSS measurements from wireless links to locate the target (e.g., intruders or victims) since a large value of variance on a wireless link infers there is a target moving in the vicinity of that particular link [2]. For instance, Wilson et. al deploy a RFbased sensor network around a residential house and use RSS sample variance during a time window to localize and track people inside the house [5]. These RSS-based systems have an implicit assumption that the target is moving at a constant speed. And thus the time window of calculating variance is selected as a fixed value. In practice, however, the target to be located will most likely moving in dynamic speeds, for example, people trapped inside a building during emergency rescue tend to run around to escape with various speeds; and intruders of an industrial facility may move at different speeds when approaching different sections of the facility. Assuming a constant moving speed when locating such targets may result in significant impact on the localization accuracy.

In this work, we first conduct a set of experiments to empirically study the impact of different moving speeds on the localization performance. We find significant performance degradation on existing RSS-based device-free localization algorithms due to the unawareness of the speed change of the moving target. To cope with dynamic moving speeds, we propose an adaptive speed change detection framework to improve localization performance over existing devicefree localization systems. Our framework consists of three components: speed change detection, determining the size of time window, and passive localization. We design two speed change detection schemes, including Average Variance Ratio (Detection-AVR), and Variance Distribution Similarity (Detection-VDS), by utilizing statistical techniques to capture moving speed changes based on information obtained from RSS measurements over wireless links. We then adaptively adjust the time-window size to facilitate accurate passive localization based on speed change detection.

The rest of the paper is organized as follows. We first provide our analysis of impact of dynamic target speed on device-free localization performance in Section II. We then 


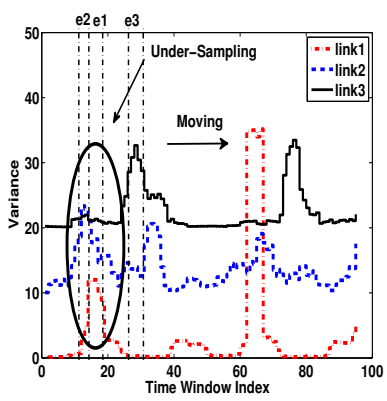

(a) Speed 1, time-window size $=1 \mathrm{~s}$

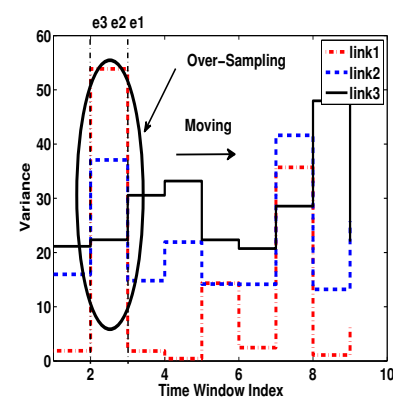

(b) Speed 2, time-window size $=3 \mathrm{~s}$

Fig. 1: Variance of signal strength in time series calculated with different time-window sizes under two different speeds, Speed 1 and Speed 2.

present our framework overview and experimental methodology used across the paper in Section III. We describe two speed change detection schemes based on statistical techniques in Section IV. In Section V, we show how to adjust time-window size adaptively to facilitate passive localization. Next, we evaluate its performance with existing representative methods in Section VI. Finally, we conclude our work in Section VII.

\section{IMPACT OF DYNAMIC TARGET SPEED ON DEVICE-FREE PASSIVE LOCALIZATION}

To understand the impact of dynamic target speed on devicefree passive localization, we experiment with different walking speeds in indoors. Specifically, we define four typical speed categories [8]: very slow speed (0-1 ft/s referred as speed 1) (e.g., an intruder breaks into an office with caution or people is hiding behind furniture in a fire building); slow speed (1$2 \mathrm{ft} / \mathrm{s}$ referred as speed 2) (e.g., walking carefully); normal speed (2-3 ft/s referred as speed 3) (walking normally); and fast speed (3-4 ft/s referred as speed 4) (e.g., walking in a hurry). Existing device-free passive localization systems using signal strength mainly capture the changes of variance of RSS measurements to determine whether a target is in the area of interest [2], [5]. Thus, the variance of RSS measurements becomes an important metric exploited in device-free localization systems. In this paper, we use the following definition of RSS variance as our basis.

We define $r_{l, W}(i)$ as the $i$ th sample of RSS on link $l$ in time window $W$, which is used to calculate variance for localization algorithms. The variance of the time window $W$ from link $l$ can be calculated as

$$
\hat{\sigma}_{l, W}^{2}=\frac{1}{N-1} \sum_{i=0}^{N-1}\left(\bar{r}_{l, W}-r_{l, W}(i)\right)^{2},
$$

where $N$ is the number of RSS samples within the timewindow size $W$, and $\bar{r}_{l, W}=\frac{1}{N} \sum_{i=0}^{N-1} r_{l, W}(i)$ is the sample mean of received signal strength within $W$. It is obvious that time window of $W$ plays a critical role when calculating RSS variance. And a larger RSS variance value of a wireless link indicates the target is close to that particular link. Note that the number of samples $N$ is related to the sampling rate and time window $W$. In our work, the sampling rate is fixed, and

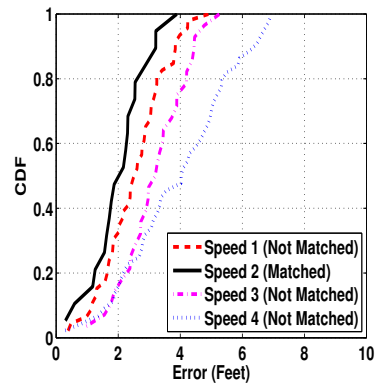

(a) Different speed, same time-window size

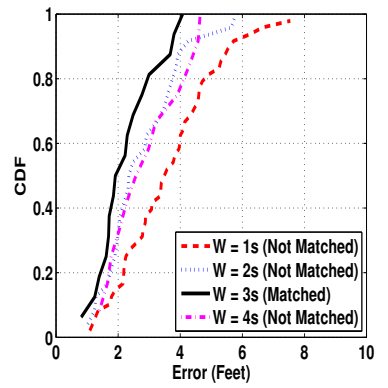

(b) Speed 1, multiple time-window sizes
Fig. 2: Device-free localization using Radio Tomographic Imaging method: (a) using the same time-window size $W=1 s$ for RSS variance calculation under four different speeds; and (b) using different time-window size for RSS variance calculation under Speed 1.

set to four packets per second. Thus, we only study the time window $W$ in the following paper.

Analysis of Variance Calculation under Different Target Speeds. To analyze the impact of different target speeds on the localization performance, we conduct experiments using 20 RFID tags in a $15 \mathrm{ft} \times 10 \mathrm{ft}$ student lab. Figure 1 presents the variance obtained from three adjacent wireless links by using two different time-window sizes when a target is walking across these three adjacent links sequentially in the order of link $1 \rightarrow$ link $2 \rightarrow$ link 3 under Speed 1 and Speed 2 respectively. When the target crosses the $i$ th link, a moving event of ' $e_{i}$ ' $(i=1,2,3)$ is derived if the change of RSS variance is above a threshold.

The correct order of moving events should be observed (i.e., $e 1 \rightarrow e 2 \rightarrow e 3)$ when the time-window size is set to $3 s$ under Speed 1, and 1s under Speed 2. However, under Speed 1, when the time-window size is set to $1 \mathrm{~s}$, the derived target moving order becomes $e 2 \rightarrow e 1 \rightarrow e 3$ as depicted in Figure 1 (a), which is inconsistent with the groundtruth. Turning to examine Speed 2, the derived target moving order becomes $e 3 \rightarrow e 2 \rightarrow e 1$ when the time-window size is $3 s$ as shown in Figure 1 (b), which is the appropriate time-window size to capture the correct order of moving events under the slower speed (Speed 1). We thus define the scenario in Figure 1 (a) as under-sampling, since the time-window size $1 s$ is too small to capture the correct target moving events for speed 1; and the scenario in Figure 1 (b) as over-sampling as the time-window size $3 s$ is too large for speed 2. This important observation indicates that appropriate time-window size needs to be chosen to accurately calculate RSS variance and consequently capture the target moving pattern.

Impact of Speed Change on Device-Free Localization Performance. We next study the impact of different speeds on device-free localization performance by using the well known RSS-based device-free localization technique, Radio Tomographic Imaging (RTI) method [9]. Figure 2 (a) shows the cumulative distribution function of the localization error when the time-window size $W$ is set to $1 \mathrm{~s}$ when the target moves in four different speeds. We find that the time-window size $1 s$ produces the best performance under Speed 2 . Whereas 
the localization process introduces large localization errors under other speeds with performance degradation of $100 \%$ on median error and $75 \%$ on maximum error under Speed 4 when the same localization algorithm RTI is applied. Similarly, when the target moves in Speed 1, we find that the localization results achieve smallest errors when the time window is set to $W=3 s$, which is inline with our observation of RSS variance changes in Figure 1 (a). For example, when comparing the performance under the time-window size of $3 \mathrm{~s}$ to that of $1 \mathrm{~s}$, we observe the median error is $80 \%$ better and the maximum error is about $100 \%$ better. The localization performance clearly show that choosing the appropriate time-window to adaptively capture the dynamic target moving pattern is critical in resulting in accurate location estimate of the target.

\section{FRAMEWORK OVERVIEW AND EXPERIMENTAL METHODOLOGY}

In this section, we provide an overview our proposed adaptive speed change detection framework and describe our experimental methodology used across the paper.

\section{A. Framework Overview}

Existing RSS-based device-free localization systems assume the target is moving under a constant speed. Thus, the localization system parameters are fixed during the localization process, making the current algorithms suffer from large performance degradation when target is moving with dynamic speeds. Our focus in this paper is to achieve high accuracy of device-free passive localization under dynamically changing speed of the target. To cope with dynamic moving speeds, there are two challenges: how to capture the speed changes, and how to adjust the localization system parameter adaptively based on the captured changes in speed? In our work, we propose an adaptive speed change detection framework to address these challenges as well as incorporate existing localization techniques for passive localization.

Our framework consists of three components as shown in Figure 3: speed change detection, determining the size of time window, and passive localization. To capture target speed changes, we design two speed change detection schemes, including Average Variance Ratio (Detection-AVR), and Variance Distribution Similarity (Detection-VDS) based on statistical learning from the information obtained from RSS measurements over wireless links. After the speed change is captured, we propose a time-window size determination scheme to adaptively update the localization system parameter, particularly the time-window size for RSS variance calculation. We note that our framework is generic which can incorporate any existing localization algorithms using signal strength, for example, the popular Radio Tomographic Imaging (RTI) method [9]. If no target speed change is detected, the localization algorithms will be applied directly with a fixed window size derived from empirical study.

\section{B. Experimental Methodology}

We conduct experiments using active RFID transmitters and receivers [10] in typical indoor multi-path environment. Each

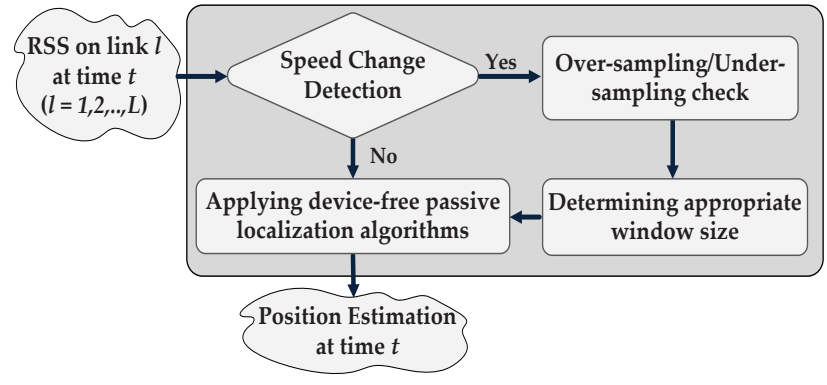

Fig. 3: Adaptive speed change detection framework overview.

RFID transmitter periodically broadcasts beacon massages with its identifier at the frequency of $900 \mathrm{MHz}$, with packet rate of four packets per second. After the receivers receive the beacon message, they extract the information of identifier together with corresponding RSS and then store it to a Linux machine that all the receivers connected to. The Linux machine, as a server, is equipped with a $2 \mathrm{GHz}$ CPU, a $1 \mathrm{~GB}$ RAM and a 120GB disk. We experiment with two different indoor environments: a small lab and a large classroom. The small lab with $15 \mathrm{ft} \times 10 \mathrm{ft}$ size is located on the first floor of Burchard building at Stevens Institute of Technology, whereas the large classroom with $45 \mathrm{ft} \times 35 \mathrm{ft}$ size is located on the first floor of Babbio center of Stevens Institute of Technology. The small lab is a microprocessor lab surrounded by chairs and shelves with electronic instruments on it, while the large classroom is equipped with tables and chairs inside it for lectures. The active RFID tags and readers are deployed as a rectangle along the walls inside the rooms at the height of $3 \mathrm{ft} 2 \mathrm{in}$. The target walks along a rectangular trace of $10 \mathrm{ft} \times 5 \mathrm{ft}$ in the small lab and $30 \mathrm{ft} \times 15 \mathrm{ft}$ in the large classroom. We experiment with 4 different speeds: Speed $1\left(\frac{5}{8} \mathrm{ft} / \mathrm{s}\right)$, Speed $2\left(\frac{15}{8} \mathrm{ft} / \mathrm{s}\right)$, Speed $3\left(\frac{5}{2} \mathrm{ft} / \mathrm{s}\right)$, and Speed $4\left(\frac{15}{4} \mathrm{ft} / \mathrm{s}\right)$. During the experiments, we change the speed using different patterns: increasing (speed $1 \rightarrow 2 \rightarrow 3 \rightarrow 4$ ), decreasing (speed $4 \rightarrow 3 \rightarrow 2 \rightarrow 1$ ), and mixture (speed $1 \rightarrow 4 \rightarrow 2 \rightarrow 3$ ).

\section{SPEED CHANGE DETECTION}

In this section, we present our speed change detection schemes including Average variance ratio (Detection-AVR), and Variance distribution similarity (Detection-VDS) scheme. We further evaluate the performance of these proposed detection schemes using real data collected in a variety of experimental scenarios.

\section{A. Speed change detection scheme}

Although the radio signal is affected by reflection, refraction, diffraction, and scattering, the RSS of the wireless links should be relatively stable if there is no movement or changes in wireless environment. However, the wireless environment will get affected and result in the changes in the RSS readings if there is people moving around. The variance of the RSS readings is thus widely used as a powerful indicator for motion detection and target localization. Furthermore, the statistic characteristic of variance of the wireless links should be different under different target speeds. In particular, the target 
with faster speed usually cuts more wireless links than that of slower one does, given a fixed time interval. Therefore, the number of links with significant variance changes under the faster speed should be more than that of the slower one.

Therefore, to detect the speed change, we compare the RSS variance in two adjacent observed time intervals: the current time interval $\Delta T$ and the previous time interval $\Delta T^{\prime}$. These two time intervals are non-overlapping with the same size. We found that the distributions of the variance are different for different speeds. Furthermore, the same speed exhibits similar variance distributions over the wireless links. Specifically, variances of larger speed produce more large values indicating more wireless links are resulted in significant variance changes under faster speed. Thus, the larger the difference of the variance distribution in these two adjacent windows is, the higher probability that the target changes its speed. This motivates us to capture the statistical changes of the observed variance as a basis to design our speed change detection schemes.

1) Average variance ratio (Detection-AVR): We define averaged variance ratio (AVR) as $\mathrm{AVR}=\frac{1}{L} \sum_{l=1}^{L} \frac{\sigma_{l, \Delta T}^{2}}{\sigma_{l, \Delta T^{\prime}}^{2}}(\mathrm{AVR}>$ 0 ), where $L$ is total number of links, $\sigma_{l, \Delta T}^{2}$ is the variance of link $l$ for $\Delta T$, and $\sigma_{l, \Delta T^{\prime}}^{2}$ is the variance of link $l$ for $\Delta T^{\prime}$. When the average variance ratio AVR falls below a threshold $\tau_{1}\left(0<\tau_{1}<1\right)$ or exceeds a threshold $\tau_{2}\left(\tau_{2}>1\right)$, we declare a speed change detected; otherwise, we declare it as no speed change. Detection-AVR is a simple scheme with lightweight computational cost that only captures coarsegrained information of variance distribution for speed change detection. We next propose Detection-VDS scheme which can capture the distribution difference of the variance in current and previous time intervals.

2) Variance distribution similarity (Detection-VDS): To further measure the variance distribution similarity for speed change detection, we utilize Kullack-leibler divergence metric [11] in Detection-VDS scheme. Given the the variance distributions over all links $P$ and $Q$ at the current time interval $\Delta T$ and previous time interval $\Delta T^{\prime}$ respectively, the KL-divergence metric is defined as $D_{K L}(P \| Q)=$ $\sum_{l} P(l) \ln \frac{P(l)}{Q(l)}$. We declare a speed change when $D_{K L}(P \| Q)$ exceeds a threshold $\varepsilon(\varepsilon<1)$; otherwise, we declare no speed change. Note that when two distributions are similar, $D_{K L}(P \| Q)$ should be close to 0 ; however, if two distributions are significantly different, $D_{K L}(P \| Q)$ is close to 1 .

\section{B. Detection Performance}

Metrics. To evaluate the performance of the proposed detection schemes, following metrics have been used. True positive is defined as declaring speed changes when there exists a speed change. False positive is defined as declaring a speed change when there is no speed changes. False negative is defined as declaring no speed changes when there exists a speed change. Detection accuracy is defined as the percentage of the trials that are correctly detected as speed change or no speed change.

Detection Accuracy. We investigate the detection accuracy

\begin{tabular}{|c|c|c|c|c|c|c|}
\hline \hline & \multicolumn{3}{|c|}{ Detection-AVR } & \multicolumn{3}{c|}{ Detection-VDS } \\
\hline$\Delta T$ & TP & FP & Acc & TP & FP & Acc \\
\hline $1 \mathrm{~s}$ & 0.85 & 0.26 & 0.795 & 0.82 & 0.18 & 0.82 \\
\hline $3 \mathrm{~s}$ & 0.84 & 0.32 & 0.76 & 0.85 & 0.22 & 0.815 \\
\hline $5 \mathrm{~s}$ & 0.81 & 0.32 & 0.745 & 0.81 & 0.21 & 0.8 \\
\hline
\end{tabular}

TABLE I: Detection Performance of our speed change detection schemes in the small lab with time interval $\Delta T=1,3,5 \mathrm{~s}$.

of our proposed speed change detection schemes by using the experimental scenario in the small lab with time interval $\Delta T=1,3,5 s$. We set scheme parameters as: $\tau_{1}=2.2$ , $\tau_{2}=0.7$, and $\varepsilon=0.6$ for small lab, and $\tau_{1}=1.8$, $\tau_{2}=0.64$, and $\varepsilon=0.55$ for large classroom. The detection results are shown in Table I. We observe that DetectionVDS scheme performs better than Detection-AVR scheme. It has the detection accuracy above $80 \%$ with around $20 \%$ false negative rate under the time interval of $1 \mathrm{~s}$ and $2 \mathrm{~s}$. Whereas Detection-AVR is around $75 \%$ accuracy. This is not surprising due to Detection-AVR only utilizes averaged variance for speed change detection without leveraging finegrained information of variance distribution. We also find that the increased detection time interval degrades the detection performance. This is because the large detection time interval flattens out the granularity of the information obtained from the RSS measurements. The larger the interval is, the more blurriness the results are. We thus choose $\Delta T=1 \mathrm{~s}$ for the rest of our study.

\section{WINDOW SIZE DETERMINATION}

In this section, we describe our window size determination scheme to adaptively update the window size for RSS variance calculation for target localization. It includes two steps: sampling check and window size determination.

Sampling Check. Once the speed change is detected, we need to update the system parameter, particularly the window size for RSS variance calculation based on the changes in speed. We first use sampling check to determine whether the previous window size for target localization encounter Undersampling or Over-sampling, as discussed in Section II. We compare the averaged variance over all the wireless links in current time window (i.e., $W$ ) and previous time window (i.e., $W-1)$. If the averaged variance in current time window is larger than that in the previous time window, we declare Oversampling indicating the window size for variance calculation in target localization should be reduced (i.e., the target is moving faster). Otherwise, it is Under-sampling, which means we need to update the window size by increasing it (i.e., the target is moving slower).

Window size determination. We utilize a binary search based method to determine the appropriate window size after sampling check. We define an indicator as $I_{W}=$ $\frac{1}{L} \sum_{l=1}^{L} \frac{\hat{\sigma}_{l, W}^{2}}{\hat{\sigma}_{l, W-1}^{2}}$ to adaptively determine the window size. For over-sampling scenario, we cautiously update the window size as $W \longleftarrow \frac{W}{2}$ (i.e., reduce the window size by half each time), till $0<I_{\frac{W}{2}}-I_{W}<\theta$. Similarly, for undersampling scenario, we cautiously update the window size as $W \longleftarrow 2 W$ (i.e., twice the window size each time), till 
$-\theta<I_{2 W}-I_{W}<0$. After window size determination, we utilize the updated window size in localization algorithms for target localization.

Performance Evaluation. The window size determination performance could be measured by examining the localization performance, which will be presented in section VI. Empirically, we set the parameter $\theta=0.3$ and 0.26 for small lab and large classroom respectively.

\section{PASSIVE Localization}

\section{A. Localization algorithms}

1) Existing Methods: We apply our speed detection scheme by incorporating two existing passive localization algorithms under our framework.

Radio Tomographic Imaging (Localization-RTI). This method develops a linear model for using RSS measurements to obtain images of moving objects. Tikhonov regularization is utilized to solve the set of linear equations formulated by the variance of RSS values. Valuable noise models are derived based on real measurements of a deployed RTI system [5].

Intersection Method (Localization-ISM). This method calculates the coordinate value of intersection from any two influential links. The weight of the two intersected influential links is defined as the sum of the RSS change of the two influential links. The final estimation is calculated as the weighted average value of all the intersection points [12].

\section{B. Performance evaluation}

We analyze the impact of speed change detection schemes on passive localization performance. We show the cumulative distribution function of the localization error, the distance between the estimated location to the true target location, when using the existing Localization-RTI method under two speed change detection schemes with time interval set to $1 s$ as shown in Figure 4 in both small lab as well as large classroom environments. We observe that localization performance has been improved largely when the speed change detection schemes are applied. Furthermore, the localization process has achieved better performance under Detection-VDS with improvement of over $30 \%$ in both median and maximum errors as compared to localization conducted without speed change detection. This is very encouraging as it indicates our speed change detection framework is highly effective to produce accurate passive localization results when target travels with dynamic speeds.

\section{CONCLUSION}

In this paper, we focus on achieving high accuracy for device-free passive localization when target is traveling in dynamic speeds. We propose an adaptive speed change detection framework with three main components: speed change detection, adaptive time-window size determination, and passive localization. Two speed change detection schemes are designed based on statistical learning from the information obtained from RSS measurements. We apply our speed detection scheme by incorporating two existing passive localization

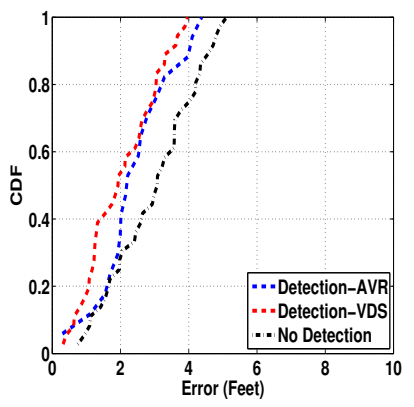

(a) small lab

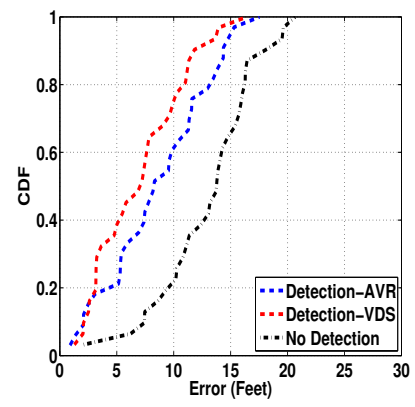

(b) large classroom
Fig. 4: Impact of speed change detection schemes on passive localization: CDF of localization error comparison when using LocalizationRTI in small lab and large classroom under two speed change detection schemes and without speed change detection.

algorithms under our framework. Extensive experiments conducted from real environments confirm the effectiveness of our proposed framework in coping with dynamic target speed by adaptively adjusting the time-window when performing passive localization. The results present significant localization performance improvements on both median and maximum errors, comparing to existing device-free localization systems treating target moving at a constant speed.

Acknowledgements: This work is supported in part by the NSF Grants CCF1018270 and CNS1217387.

\section{REFERENCES}

[1] M. Youssef, M. Mah, and A. Agrawala, "Challenges: device-free passive localization for wireless environments," in the 13th annual ACM international conference on Mobile computing and networking, 2007.

[2] J. Yang, Y. Ge, H. Xiong, Y. Chen, and H. Liu, "Performing joint learning for passive intrusion detection in pervasive wireless environments," in IEEE International Conference on Computer Communications (INFOCOM), 2010.

[3] C. Xu, B. Firner, Y. Zhang, R. Howard, J. Li, and X. Lin, "Improving rfbased device-free passive localization in cluttered indoor environments through probabilistic classification methods," in the 11th international conference on Information Processing in Sensor Networks, 2012.

[4] J. Yang and Y. Chen, "Indoor localization using improved rss-based lateration methods," in IEEE Global Communications Conference (GLOBECOM), 2009

[5] J. Wilson and N. Patwari, "See-through walls: Motion tracking using variance-based radio tomography networks," IEEE Transactions on Mobile Computing, 2011.

[6] C. Chang and A. Sahai, "Object tracking in a 2D UWB sensor network," in the Thirty-Eighth Asilomar Conference on Signals, Systems and Computers, Nov. 2004.

[7] T. Pratt, S. Nguyen, and B. Walkenhorst, "Dual-polarized architectures for sensing with wireless communications signals," in IEEE Military Communications Conference., Nov. 2008.

[8] J. A. H. Raymond C. Browning, Emily A. Baker and R. Kram, "Effects of obesity and sex on the energetic cost and preferred speed of walking," Applied Physiology, 2005.

[9] J. Wilson and N. Patwari, "Radio tomographic imaging with wireless networks," IEEE Transactions on Mobile Computing, vol. 9, pp. 621632, May 2010.

[10] B. Firner, S. Medhekar, Y. Zhang, R. Howard, and W. Trappe, "Pip tags: Hardware design and power optimization," in the Fifth Workshop on Embedded Networked Sensors, 2008.

[11] H. Zhu, "On information and sufficiency," Annals of Mathematical Statistics, 1951.

[12] D. Zhang, J. Ma, Q. Chen, and L. M. Ni, "An RF-based system for tracking transceiver-free objects," in the Fifth IEEE International Conference on Pervasive Computing and Communications, 2007. 